\title{
Physically-based modeling and animation of tree branch patterns
}

\author{
Meng Yang $•$ Bin Sheng $\cdot$ Enhua Wu $\cdot$ Hanqiu Sun
}

Published online: 8 December 2009

(C) Springer-Verlag 2009

Due to copyright violation by the students, this article was retracted.

M. Yang

Inst. of Software, Chinese Academy of Sciences, Beijing 100190,

China

e-mail: yangm@ios.ac.cn

B. Sheng $(\bowtie) \cdot$ H. Sun

Department of Computer Science and Engineering, The Chinese

University of Hong Kong, Hong Kong, China

e-mail: bsheng@cse.cuhk.edu.hk

H. Sun

e-mail: hanqiu@cse.cuhk.edu.hk

E. $\mathrm{Wu}$

University of Macau \& Inst. of Software, Chinese Academy

of Sciences, Macau, China

e-mail: ehwu@umac.mo 